\title{
Mecânica Relacional: A Propósito de uma Resenha
}

\author{
Relational Mechanics: Concerning a book review \\ O. Escobar \\ Instituto de Física Gleb Wataghin \\ Universidade Estadual de Campinas, UNICAMP \\ 13084-971 - Campinas, SP, Brazil \\ V. Pleitez \\ Instituto de Física Teórica \\ Universidade Estadual Paulista \\ Rua Pamplona, 145, 011405-900, São Paulo, SP, Brasil
}

Recebido em 19 de Março 2001. Aceito em 25 de Junho 2001

\begin{abstract}
Neste artigo fazemos uma análise crítica à proposta da Mecânica Relacional tal como apresentada no livro de mesmo nome, objeto de uma resenha recente nesta revista.
\end{abstract}

We present a critical analysis of what is called Relational Mechanics, as it has been presented in a book thus entitled, which has been recently reviewed in this journal.

\section{Introdução}

Ainda que não seja parte do dia-a-dia de um pesquisador, de vez em quando a questão do método científico aparece para ser considerada, mesmo que seja de maneira breve, instantânea. No entanto, seja motivado pela leitura de um trabalho exótico colocado na rede eletrônica de preprints, seja pelo artigo confuso de uma revista especializada, vez ou outra somos levados a nos perguntar: $\mathrm{O}$ que distingue a ciência de outras atividades? Como fazem os cientistas para eliminar ou confirmar as teorias? É possível distinguir ciência da pseudociência? Existe ciência patológica? Por exemplo, este tipo de preocupação teria alguma implicação na nossa vida de pesquisador? É (ou deve ser) a ciência conservadora? É freqüente lembrar dos casos de perseguição científica: Giordano Bruno, Galileu ou, de pelo menos cegueira coletiva da comunidade científica: Boltzmann por exemplo ou, mais recentemente, Alfred Wegener [AL88]. Deveria isso imobilizar a comunidade científica? Afinal quem é essa comunidade? ${ }^{1}$

A maioria das atividades que podemos classificar com os adjetivos adicionais ao substantivo ciência, mencionados no parágrafo anterior, são realizadas fora das universidades. Assim, com exceção de alguns cientis- tas como C. Sagan [SA96], os pesquisadores não se dão o trabalho de discutir e criticar essas atividades da mesma maneira como criticam os próprios trabalhos científicos. Afinal, uma das características do dia-adia da ciência é essa tensão entre propostas alternativas como explicação dos fenômenos naturais. Mas, e quando isso acontece numa universidade? São as críticas necessárias? Violariam a liberdade acadêmica? A liberdade acadêmica deve ser ampla e irrestrita? Se sim, é isso compatível com um bom critério de utilização dos fundos públicos?

Recentemente foi publicado o livro Mecânica Relacional (MR) [AK99]. Nesse livro pretende-se colocar uma nova visão da mecânica. Seria mais um livro de ensino dessa disciplina ou um livro de divulgação científica? Nenhum desses casos, senão vejamos. Um livro que afirme no prefácio:

Este livro tem como objetivo apresentar as propriedades e caraterísticas desta nova visão da mecânica [...] fica fácil fazer uma comparação com as visões anteriores ${ }^{2}$ do mundo (newtoniana e einsteiniana), ${ }^{3}$

\footnotetext{
${ }^{1}$ Estes casos não são exatamente como os manuais descrevem mas não é nosso objetivo aqui dar detalhes deles.

${ }^{2}$ Os negritos são nossos.

${ }^{3}$ Observe-se o tempo passado com relação a física newtoniana e einsteiniana.
} 
e que, além disso, é editado pelo Centro de Lógica, Epistemologia e História da Ciência da UNICAMP não pode passar desapercebido pela comunidade científica do país. Ele deve ser analisado, comentado, criticado pelos cientistas da mesma forma que o são as teorias e resultados experimentais da ciência normal. Não é possível que alguém chegue dizendo que as visões de Newton e Einstein estão erradas e ninguém da comunidade dos físicos diga nada. Confirme-se e aceite-se seu impacto na física e ciências afins ou coloque-se esta obra em merecido ostracismo. Esperamos deixar claro neste artigo que, corretamente analisado, o assunto levantado pelo livro em questão sequer polêmico é. Porém, depois das características acima mencionadas, o livro tem de ser analisado criticamente. Também, acrescente-se, de maneira definitiva.

É necessário saber se de fato representa uma visão nova da física porque, se for verdade teríamos que rever tudo que foi feito nas últimas décadas, vários prêmios Nobel deviam ser devolvidos. Mas, e se não fosse? Seria um exemplo de ciência patológica? Enfim ... definitivamente não pode passar sem ser percebido, ainda que não seja polêmico.

Já foi feita uma resenha sobre o referido livro, publicada nesta revista [SO99], daí a "resenha" do título. Nessa resenha não se poupam elogios à nova visão da física pretendida no livro MR. No entanto, é interessante notar que na versão publicada dessa resenha foi acrescentada uma nota de rodapé onde se agradece a um árbitro anônimo, o qual pedia para o autor da resenha ler o livro de A. Pais [PA95]. Nesse livro encontrase uma história mais detalhada sobre a influência do princípio de Mach no pensamento de Einstein. No entanto, a leitura do livro de Pais deveria ter induzido o autor da resenha a revê-la toda e mesmo mudar sua opinião sobre o livro. Mas, no próprio livro MR, apenas são citadas as palavras de Einstein sobre a influência que Mach teve sobre ele num certo período de sua vida. Omitem-se outras, e que nós incluimos aqui, nas quais Einstein revê a sua posição com relação ao princípio de Mach. ${ }^{4}$

Assim, é nosso objetivo fazer uma crítica à proposta da MR baseada nas teorias científicas desenvolvidas nos últimos 100 anos, mais ou menos. Tentamos deixar claro para o leitor que: 1) não é verdade que as teorias da relatividade especial e geral (TRE e TRG, respectivamente) estejam erradas, elas são de fato muito bem verificadas experimentalmente; além do que, conceitualmente elas têm permitido avanços técnicos e teóricos em diversas áreas como a astronomia, a astrofísica e, principalmente, na área da física das interações fundamentais. 2) É sim a MR que não descreve os fenômenos naturais observados.

Nosso objetivo não é convencer o autor do livro que a sua proposta não concorda com os dados experimentais, mas procurar convencer o leitor, que por pouca familiaridade com as teorias da física do Sec. XX pode pensar estar diante de uma proposta que na sua opinião é, na pior das hipóteses, pelo menos "científica", perceber por si só que o que nós colocamos aqui é correto: não só as críticas às TRE e TRG estão erradas, mas a própria MR está há muito tempo descartada pela experiência.

Claro, não esperamos que apenas a leitura desta resenha seja suficiente para tal efeito. Será necessário que o leitor que ainda tiver dúvidas procure consultar algumas das referências aqui citadas que poderão ser-lhe de utilidade, ainda que não pretendamos ser exaustivos nesse aspecto.

Na Sec. II revisamos o princípio de Mach visando esclarecer qual foi a sua influência sobre Einstein. Ficará claro que a partir de certo momento Einstein afastouse dele. Verifica-se também que esse princípio não seria necessário para a elaboração daquelas teorias (TRE e TRG). Na Sec. III enfatizamos que as teorias da relatividade, especial e geral, são teorias bem estabelecidas experimentalmente e que não procedem as críticas a ambas feitas no livro MR [AK99]. Pelo contrário, mostramos na Sec. IV que a MR é a teoria que está errada. Alguns comentários finais estão na Sec. V.

\section{O princípio de Mach}

Ernst Mach (1838-1916) foi um cientista polivalente, mas a sua maior influência foi na mecânica de fluidos e na filosofia. Foi um crítico do conceito de espaço absoluto da mecânica newtoniana. No prefácio da primeira edição (alemã) do seu livro disse [MA83]

O presente volume não é um tratado sobre a aplicação dos principios da mecânica. Seu propósito é esclarecer idéias, expor o significado real dessa matéria, e liberá-la do obscurantismo metafísico.

Essas palavras devem ser entendidas no contexto do empirismo radical que muitos cientistas defendiam nas últimas décadas do Sec. XIX. A termodinâmica era então rainha absoluta como paradigma de ciência. Estava baseada apenas em quantidades que podiam ser medidas no laboratório, outro tipo de abordagem era considerado metafísico. Isso influenciaria muito o pensamento de Einstein, mas depois ele aceitou que "é a teoria que diz o que é observável e o que não é" [HE78].

A. Pais, referindo-se à critica que Mach fizera em seu livro de 1883 [MA83] à mecânica de Newton, disse [PA95a]

As mencionadas referências mostram que Mach reconhecia claramente os aspectos clássicos da mecânica clássica e que não

\footnotetext{
${ }^{4}$ Deveríamos dizer, em geral, da filosofia de Mach.
} 
esteve longe de exigir uma teoria da relatividade geral, isto há cerca de meio século antes!

Porém Mach disse em $1913^{5}$

Devo $[\cdots]$ com igual intensidade recusar ser precursor dos relativistas, como me retirei da crença atomista da atualidade.

A visão de Mach da mecânica está bem resumida na afirmação de que

quando $[\cdots]$ afirmamos que um corpo conserva sem alteração sua direção e velocidade no espaço, nossa afirmação não é nem mais nem menos do que uma referência abreviada ao universo inteiro (os itálicos são de Mach) $[\cdots]$

Comentando as palavras de Mach acima Pais disse [PA95b]:

Não encontramos no livro de Mach como se manifesta esta importância de todos os corpos, pois ele nunca propôs um esquema dinâmico explícito para esta nova interpretação da lei de inércia.

Isso ainda é verdade: o princípio de Mach não foi implementado de maneira consistente por nenhuma teoria. O conhecido astronômo $\mathrm{H}$. Bondi é bem claro a respeito [BO68]

Ainda que o Principio de Mach estivesse correto, é necessário considerar outras teorias para levar em conta as evidências experimentais e observacionais [...] o postulado da relatividade da inercia (Principio de Mach) é intelectualmente agradável de muitas maneiras, e parece a alguns autores que deve ser inescapavelmente verdadeiro. Outros o vêem com suspeita, dado que não tem sido possível até agora expressá-lo em forma matemática (nem mesmo na relatividade geral), e uma vez que não tem sido possível verificá-lo experimentalmente.

Como dissemos antes, a construção da TRE foi muito influenciada pela filosofia pragmática de Mach: foram usadas apenas quantidades passíveis de serem medidas. Einstein posteriormente também se afastou dessa filosofia, mas não consideraremos isso aqui. Por outro lado, o mesmo aconteceu com a TRG. Em 1912, usando uma versão rudimentar da teoria da gravitação, Einstein mostrou que se uma esfera oca massiva é acelerada em torno de um eixo que passa pelo centro no qual se encontra uma massa inercial pontual, então a massa inercial desta última é aumentada. Nas próprias palavras de Einstein [PA95c]

Esta [conclusão] fornece plausabilidade à conjectura de que a inércia total de um ponto com massa é um efeito que decorre da presença de todas as outras massas, graças a um tipo de interação com estas últimas $[\cdots]$. É este justamente o ponto de vista sustentado por Mach nas suas investigações profundas sobre este tema.

Vemos que Einstein tinha o princípio de Mach como guia para a construção das teorias da relatividade. ${ }^{6}$

Em 1917 Einstein, no que seria o primeiro trabalho da história sobre cosmologia relativista [EI17], ainda pensava de acordo com a citação acima a respeito das idéias de Mach [PA95c]. Ele ainda tentava implementar uma origem inteiramente material da inércia, isto é, que a métrica $g_{\mu \nu}$ do espaço-tempo seria determinada apenas pela matéria [PA95c]. De fato, nesse trabalho Einstein introduz o "termo cosmológico" para estar em acordo com o princípio de Mach, isto é, para ter um universo fechado, e também para conseguir um universo homogêneo, isotrópico e estático e tal que $g_{\mu \nu}=0$ na ausência de matéria.

Provavelmente a demonstração de de Sitter em 1917 sobre a existência de soluções das equações da TRG no vácuo: $g_{\mu \nu} \neq 0$ e $T_{\mu \nu}=0$, isto é, soluções para as equações da TRG sem matéria (que Einstein acreditava não existirem) é que começou a minar sua credibilidade nesse princípio. A outra motivação, de um universo homogêneo, isotrópico e estático, foi eliminada quando em 1922 A. Friedmann demonstra que era possível um universo homogêneo e istrópico se ele estivesse se expandindo (e não estático como supunha Einstein). Mas o acontecimento crucial foi então a descoberta de de Sitter que as equações de Einstein com o termo cosmológico tinham solução mesmo no vazio: a inércia é diferente de zero mesmo sem a presença da matéria. Inicialmente Einstein, que antes tinha dito que "um corpo num universo vazio não poderia ter inércia", objetou a solução de de Sitter mas logo ele se convenceu que aquele tinha razão. Não era mais possível que $g_{\mu \nu}$ pudesse ser determinado completamente pela matéria. Vemos então que não se pode fazer uma citação de Einstein de 1917 sem levar em conta que alguns anos depois ele se convenceria de seu próprio erro!

Segundo Pais [PA95f]

Anos mais tarde, o entusiasmo de Einstein pelo princípio de Mach esmoreceu e, finalmentem, desapareceu.

Por exemplo, em 1954 em uma carta a Felix Pirani ele disse [HO82, PA95d]

\footnotetext{
${ }^{5}$ Esta frase está traduzida de maneira diferente por diferentes autores. Aqui queremos somente lembrar a intransigência de Mach, sendo que ele mesmo acreditava ser "não dogmático".

${ }^{6}$ De fato foi Einstein quem chamou a conjetura da origem da inércia de Mach como o "Princípio de Mach".
} 
$\mathrm{Na}$ minha opinião nunca mais deveríamos falar do princípio de Mach. Houve uma época na qual pensava-se que os 'corpos ponderáveis' eram a única realidade física e que, numa teoria todos os elementos que não estiverem totalmente determinados por eles, deveriam ser escrupulosamente evitados. Sou consciente que durante um longo tempo também fui influenciado por essa idéia fixa.

Pouco tempo depois ele disse [SC49]

...Assim, se consideramos como possível, campos gravitacionais de extensão arbitrária os quais não estão inicialmente espacialmente restringidos, o conceito de 'aceleração relativa ao espaço' perde seu significado e com ele o princípio da inércia junto com todo o problema de Mach.

Em geral os cosmólogos aceitam o ponto de vista posterior de Einstein, por exemplo, segundo Bondi [BO68]

por esta razão ele introduziu a chamada constante cosmológica na esperança de reconciliar a relatividade geral com o principio de Mach […] Esta esperança não foi, contudo, realizada.

A origem da inércia (das massas) continua a ser um ponto em aberto em qualquer teoria fundamental das partículas elementares. Assim segundo Pais [PA95d]:

Do meu ponto de vista, até agora o princípio de Mach não fez avançar decisivamente a física, e a origem da inércia é, e continua a ser, o assunto mais obscuro na teoria de partículas e campos. O princípio de Mach pode, conseqüentemente ter futuro, mas não sem a teoria quântica.

Podemos concluir que o princípio de Mach não foi até agora confirmado nem teórica nem experimentalmente. Que todas as teorias atuais não tenham sido capazes de implementá-lo pesa mais contra ele que contra as próprias teorias. O estudo da influência de Mach sobre Einstein pertence mais ao que Holton chama de "a peregrinação filosófica de Albert Einstein" [HO82b]

uma peregrinação a partir de uma filosofia da ciência na qual o sensacionalismo e o empirismo ocupavam uma posição central, até outra fundada no realismo racional.

Definitivamente então, a partir de um certo momento, Einstein e outros físicos bem conhecidos não levaram mais em conta o princípio de Mach como guia na construção das suas teorias.

\section{As Teorias da Relatividade estão erradas?}

Não. Muito pelo contrário. Vide, por exemplo, o amplo artigo de Will [WI79] e seu mais recente livro [WI96], onde se resume os testes experimentais de ambas teorias da relatividade, a especial e a geral. No caso da relatividade especial, que é sem dúvida a melhor testada, Will diz [WI79b]:

Uma grande quantidade de experiências em laboratórios de altas energias têm verificado a validade da teoria de relatividade especial no limite quando os efeitos gravitacionais podem ser ignorados. Estes experimentos vão de testes diretos da dilatação do tempo a teste esotérico das predições da teoria quântica de campos relativista.

No entanto o autor de MR insiste [AK99b]:

Defendemos aqui que as teorias de Einstein não implementaram as idéias de Mach e que a Mecância Relacional é uma teoria melhor do que as de Einstein para descrever os fenômenos observados na natureza $[\cdots]$ Einstein e seus seguidores criaram muitos problemas com esta teoria.

A TRE tornou a hipótese do éter supérflua, não mostrou que este não existia. Isso é típico do conhecimento científico. $\mathrm{O}$ autor do texto MR não entendeu como funciona a ciência. A ciência não mostra que os deuses da chuva e anjos carregando os planetas não existem. Ela apenas não usa essas hipóteses. Claro, se acreditamos que existe uma realidade independente de nós mesmos e que é, pelo menos parcialmente, desvendada pela ciência, então o fato de o éter não ser necessário para as teorias físicas pode ser interpretado como indicativo de sua inexistência.

As críticas do autor à TRE não são corretas e mostram a pouca familiaridade dele com o tema. Por exemplo [AK99d]

...há muitos problemas com as teorias da relatividade especial e geral. Enfatizamos alguns aqui.

1) elas são baseadas na formulação de Lorentz da eletrodinâmica de Maxwell, formulação que apresenta diversas assimetrias como as apontadas por Einstein e muitos outros [... Há uma teoria do eletromagnetismo que evita todos estas assimetrias de forma natural $[\cdots]$ a eletrodinâmica de Weber... 
É sabido, faz mais de 100 anos, que a eletrodinâmica de Weber não é uma descrição dos fenômenos eletromagnéticos: na sua versão original não prevê a existência de ondas eletromagnéticas! Da maneira como é comparada com "a formulação de Lorentz da eletrodinâmica de Maxwell" parece que a de Weber é uma outra formulação da mesma. A formulação de Lorentz a que se refere o autor é a das equações de Maxwell microscópicas. Nela todos os fenômenos eletromagnéticos podem ser vistos como sendo produzidos por portadores de cargas elementares como os elétrons e os núcleos atômicos. As equações de Maxwell macroscópicas podem, em casos simples, ser deduzidas a partir das equações de Maxwell-Lorentz. Na verdade é a formulação de Lorentz que é generalizável para a mecânica quântica relativista.

A assimetria a que se refere o autor é aquela mencionada no primeiro artigo de Einstein de 1905 sobre a TRE [EI05, EI05b]:

Como é sabido, a Eletrodinâmica de Maxwell-tal como atualmente se concebeconduz, na sua aplicação a corpos em movimento, a assimetrias que não parecem ser inerentes aos fenômenos. 7 Consideremos, por exemplo, as ações eletrodinâmicas entre um ímã e um condutor. $\mathbf{O}$ fenômeno observável depende unicamente do movimento relativo do condutor e do ímã, ao passo que, segundo a concepção habitual, são nitidamente distintos os casos em que o móvel é um, ou outro, desses corpos. Assim, se for móvel o ímã e o condutor estiver em repouso, estabelecerse-á em volta do ímã um campo elétrico com determinado conteúdo energético, que dará origem a uma corrente elétrica nas regiões onde estiverem colocadas porções do condutor. Mas, se é o ímã que está em repouso e o condutor que está em movimento, então, embora não se estabeleça em volta do ímã nenhum campo elétrico, há no entanto uma força eletromotriz que não corresponde a nenhuma energia, mas que dá lugar a correntes elétricas de grandeza e comportamento iguais às do primeiro caso, produzidas por forças elétricas-desde que, nos dois casos considerados, haja identidade no movimento relativo.

Mais adiante, depois de apresentar a sua teoria, Einstein diz [EI05c]

Como se vê, na teoria que se desenvolveu, a força eletromotriz apenas desempenha o papel de conceito auxiliar, que deve a sua introdução ao fato de as forças elétricas e magnéticas não terem existência independente do estado de movimento do sistema de coordenadas.

É também claro que a assimetria mencionada na introdução, que surge quando se consideram as correntes elétricas provocadas pelo movimento relativo de um íman e de um condutor, desaparece agora.

Vemos então que o autor da MR não entendeu o argumento de Einstein no seu artigo de 1905. Hoje diríamos que as equações de Maxwell, usando a notação de 3-vetores, introduzida por Heaviside, não são manifestamente invariantes sob as transformações de Lorentz. Mas essa assimetria não ocorre, como observado pelo próprio Einstein, nos fenômenos observados, como sabemos desde Faraday. A assimetria desaparece porque no sistema de referência que acompanha o condutor, do ponto de vista da TRE, temos também um campo elétrico: $\vec{E}^{\prime} \propto v \times \vec{B}^{\prime}$. A inter-relação entre campos elétricos e magnéticos na eletrodinâmica de Maxwell só foi descoberta por Einstein. Ainda que a teoria microscópica (que é de Lorentz mas continua sendo a eletrodinâmica de Maxwell) seja relativisticamente invariante, o fenômeno mencionado foi percebido por Einstein mesmo. Assim, é apenas quando se descobre a invariância das equações de Maxwell sob transformações de Lorentz que a assimetria desaparece. Isso está bem explicado em livros básicos como o de Purcell [PU78] apenas para dar um exemplo.

Outro ponto que deve ser enfatizado é que o autor da MR não compreendeu a covariância geral, confundindoa com a covariância introduzida por Minkowski que se refere apenas às transformações de Lorentz [AK99j]. Na TRG sim, temos uma covariância geral, no sentido que as equações são as mesmas em qualquer sistema de referência, inercial ou não.

Como já foi dito acima, a TRE não é verificada somente pelas experiências diretas. Todo o edifício conceitual da física de partículas elementares e as suas técnicas teóricas e experimentais estão baseados nela. Mesmo que alguém mostrasse que as experiências clássicas não são suficientes para testar com a precisão necessária a teoria, esta não seria facilmente abandonada porque já foi confirmada na prática em outras áreas.

O mesmo ocorre com a TRG: nos anos de 193940 Einstein, com Leopold Infeld e Banesh Hoffmann, tratou o problema do movimento de $N$ corpos com a relatividade geral. Segundo Misner et al. [MI73]

As equações […] se chamam equações de Einstein-Infeld-Hoffman (EIH) para a geometria e evolução de um sistema de muitos corpos. Elas são usadas na análise das órbitas planetárias no sistema solar. Por exemplo, o Caltech Jet Propulsion Laboratory as usa, numa forma modificada, para

\footnotetext{
${ }^{7}$ Os negritos são nossos.
} 
calcular as efemérides para observar os planetas e as naves espaciais.

Vemos então que já existem aplicações da TRG (ver mais sobre isso mais adiante).

Além disso novos testes mais acadêmicos são obtidos. Por exemplo, em 1993 R. A. Hulse e J. H. Taylor ganharam o prêmio Nobel de Física: "pela descoberta de um novo tipo de pulsar, uma descoberta que abriu novas possibilidades para o estudo da gravitação." [NO93].

Mas o que isso significa? Bem, Hulse e Taylor observaram durante quase 20 anos, de 1975 a 1993, um pulsar binário com o exótico nome de PSR $1913+16^{8}$ e que consiste de um par de estrelas de nêutrons, com um raio de algumas dezenas de quilômetros, com massa da ordem da massa do Sol e com uma distância relativa da ordem da algumas vezes a distância Terra-Lua girando ao redor de seu centro de massa. Eles determinaram que a perda de energia do sistema era consistente com os cálculos baseados na teoria da relatividade geral [PE98]. Este foi um teste de TRG mais definitivo que os três testes clássicos: o perihélio de Mercúrio, o desvio da luz pelo Sol e o atraso de relógios em campos gravitacionais. Estes testes estavam restritos ao nosso sistema solar onde o campo gravitacional é fraco. Os resultados de Hulse e Taylor foram os primeiros testes de grande precisão da TGR. Segundo a TRG, objetos em órbita, como no caso do pulsar acima mencionado, irradiam energia sob a forma de ondas gravitacionais (ondulações no espaço-tempo). Isto implica numa perda de energia do sistema que pode ser calculada usando a TRG. Os resultados de Hulse e Taylor concordaram muito bem (é um número da ordem de $10^{-14}$ e medido com uma precisão de $0.5 \%$ !) com as previsões teóricas da TGR.

Em 100 anos de prêmios Nobel apenas em 6 ocasiões não foi entregue. Deste total, 27 estão relacionados de alguma maneira com a relatividade especial e pelo menos 1 com a TGR. Estes são: P. A. M. Dirac (1933, teoria relativista do elétron), J. Chadwick (1935, descoberta do nêutron), C. D. Anderson (1936, descoberta da anti-matéria); E. O. Lawrence (1939, invenção do ciclotron); W. Pauli (1945, princípio de exclusão); H. Yukawa (1949, pelo mésons $\pi$ ); J. D. Cockcroft e E. T. S. Walton (1951, aceleradores de partículas); W. E. Lamb e P. Kush (1955, efeitos relativísticos nos átomos); C. N. Yang e T. D. Lee (1957, violação da paridade); E. G. Segré e O. Chamberlein (1959, descoberta de anti-matéria hadrônica: anti-próton); E. P. Wigner (1963, princípios de simetria); S-I. Tomonaga, J. Schwinger e R. Feymann (1965, pela eletrodinâmica relativista); H. A. Bethe (1967, pelos mecanismos relativistas da criação da energia nas estrelas); L. W. Alvarez (1968, descobertas experimentais em física de partículas elementares); M. Gell-Mann (1969, contri- buições teóricas à física de partículas elementares); B. Richter e S. C. C. Ting (1976, descoberta do quark b); S. Glashow, A. Salam e S. Weinberg (1979, modelo de interações eletrofracas); J. W. Cronin e V. L. Fitch (1980, descoberta da violação da simetria discreta CP); S. Chandrasekhar (1983, evolução e estrutura das estrelas) C. Rubbia e S. van der Meer (1984, descoberta dos bósons intermediários $\left.W^{ \pm}, Z^{0}\right)$; L. M. Lederman, M. Schwartz e J. Steinberg (1988, descoberta do segundo neutrino, $\left.\nu_{\mu}\right)$; G. Charpac (1992, detetores de partículas relativistas); M. Perl e F. Reines (1995, descobertas do lépton $\tau$ e deteção do neutrino do eletron, $\nu_{e}$, respectivamente); G. 't Hooft e M. J. G. Veltman (1999, correções quânticas ao modelo eletrofraco de Glashow-Salam-Weinberg). Todas estas descobertas teóricas ou experimentais somente têm sentido no contexto de teorias quântico-relativistas. Com relação à TGR podemos colocar os já acima mencionados R. A. Hulse e J. H. Taylor (1993, deteção indireta de ondas gravitacionais). Não mencionamos aqui alguns resultados também premiados que de maneira indireta usam a eletrodinâmica de Maxwell, que sendo relativista poderia ser considerada como teste indireto da TRE. Se as teorias da relatividade estivessem erradas todos esses prêmios Nobel teriam que ser devolvidos. O leitor interessado pode visitar a página WWW da Fundação Nobel [NO00].

Com relação à dilatação do tempo nas TRE e TRG, o astrônomo real Martin Rees diz que mesmo não sendo perceptível nos movimentos e tempos do dia-adia [RE00]

Esse pequeno efeito [dilatação do tempo da TRE] foi agora, contudo, medido por experimentos com relógios atômicos com precisão de um bilionésimo de segundo, e está de acordo com as previsões de Einstein... uma "dilatação do tempo" semelhante é causada pela gravidade: nas proximidades de uma grande massa, os relógios tendem a andar mais devagar.... essa dilatação deve ser levada em conta, juntamente com os efeitos do movimento orbital, na programação do notavelmente preciso sistema GPS (Global Positioning Satellite)...

De fato, atualmente o sistema GPS tem uma precisão de milimetros, uma discordância de uma milhonésima de segundo implica num erro da ordem de 300 metros! [HE96].

Além disso as cada vez mais precisas medidas do fator $(g-2)_{\mu}$ são compatíveis com dilatações da vida média do múon de até $\gamma=29.3$ [BR01] Isso mostra que o efeito nos múons não apenas os observados na atmosfera e o argumento do autor da MR não se sustenta (ver a próxima seção).

\footnotetext{
${ }^{8}$ PSR significa "pulsar" e 1913+16 especifica a posição do pulsar no céu.
} 
Poderíamos mencionar outras situações onde fica claro o pouco conhecimento que o autor de MR tem das teorias da relatividade. Bastam mais uns exemplos: o autor da MR não sabe que não existe "paradoxo dos gêmeos", e não entendeu o atraso do relógio [AK99l]. Não comentamos mais sobre este ponto porque é bastante bem considerado em livros elementares de relatividade [GE78].

\section{Está a Mecânica Relacional errada?}

Sim. Após criticar a TRG de Einstein por não ter implementado a rota de construir a teoria apenas em termos de distâncias relativas, diz [AK99c]

... como veremos neste livro, é possível seguir esta rota com sucesso utilizando uma lei de Weber para a gravitação.

Por que usar uma lei da gravitação baseada numa lei da eletrostática que não deu certo? Mesmo que alguém acredite na MR, dever-se-ia perguntar: por que essa força e não outra? Assim, existiriam tantas MR quanto possíveis autores. O papel desempenhado pelas simetrias nas leis da Física não foi comprendido pelo autor da MR: ele ignora os trabalhos de cientistas como E. Wigner, H. Weyl, C. N. Yang etc! As simetrias têm desempenhado um papel importante na descoberta de novas leis da natureza, mas na MR lemos que tudo isso não é necessário na nova física ali proposta! No momento que se abre mão dos princípios de simetria tudo é válido!

A MR está baseada em três postulados. Os dois primeiros são compatíveis com as leis de Newton. Já o terceiro postulado, diz [AK99e]

A soma de todas as forças de qualquer natureza (gravitacional, elétrica, magnética, elástica, nuclear,...) agindo sobre qualquer corpo é sempre nula em todos os sistemas de referência.

Bom, sabemos que cada uma das forças mencionadas no postulado III tem uma intensidade característica bem diferente. Por exemplo, a força gravitacional é $10^{-40}$ vezes mais fraca que a força eletromagnética. Assim, se a sua soma se anula, então devem existir outras forças tais que façam a soma ser zero. Onde estão essas forças?

Compare com o postulado de Einstein "a luz, no espaço vazio, se propaga sempre com uma velocidade determinada, independente do estado de movimento da fonte luminosa".

Na melhor das hipóteses, em 1905 estes dois postulados poderiam ter sido considerados como alternativas possíveis. Hoje, depois de tantos testes experimentais e teóricos, não mais.

Mas, na MR se insiste na eletrodinâmica de Weber, por exemplo [AK99f]

As propriedades e vantagens da teoria eletromagnética de Weber foram consideradas em outro livro.

Essa teoria não tem nenhuma vantagem, ela já foi descartada como proposta científica. Enfatizamos, um século de experimentos e aplicações tecnológicas e, não menos importante, os esquemas conceituais construídos a partir da eletrodinâmica de Maxwell não deixam espaço para ela. Lembre-se disso, caro leitor, quando assistir televisão ou ouvir a sua música favorita no seu $C D$ player.

Da eletrodinâmica se passa à gravitação, o autor continua

... em analogia à eletrodinâmica de Weber, propomos como a base para a mecânica relacional que a lei de Newton da gravitação universal seja modificada para ficar nos moldes da lei de Weber.

O leitor deve se convencer por ele mesmo que teorias de campo não relativísticas não estão de acordo com a experiência. O deslocamento Lamb e o momento magnético do elétron e do múon são exemplos, entre outros, da validade dessas teorias. ${ }^{9}$

Mais ainda, os testes mais fortes, repetimos, de uma teoria são os indiretos. Por exemplo, toda a física de aceleradores não seria possível sem a TRE. Como foi mencionado na seção anterior, mesmo o sistema GPS está usando ambas TRE e TRG. Como explicar esse sucesso no contexto da MR?

O fato que os testes indiretos passam a ser mais importantes que os diretos (que são importantes quando se está propondo uma teoria) faz com que caso alguém hoje repetisse as experiências de Michelson-Morley ou Fizeau e afirmasse ter achado resultados opostos aos das experiências originais, o experimento será encarado como errado! [De fato isso aconteceu com a experiência de Michelson-Morley: em 1926 um físico chegou a conclusões opostas. Nunca se confirmou onde estava o erro mas já não era mais necessário achá-lo!]. É isso que quer dizer o conhecido físico, premio Nobel de 1977, P. W. Anderson quando afirma que [AN90]

É da natureza da física que suas generalizações são continuamente testadas não apenas por cuidadosos experimentos diretos mas usualmente mais severamente 10 pela consistência total de toda a estrutura da física. A moral finalmente é que a físicade fato a ciência como um todo- é um fina rede sem remendos.

\footnotetext{
${ }^{9}$ Estes são os cálculos esotéricos de teoria quântica de campos mencionada por Will acima.

${ }^{10}$ Os negritos são nossos.
} 
A MR é uma teoria de tempo absoluto e não passa por testes que evidenciam a "dilatação do tempo". E mais, os argumentos (fracos) contra a TRE e TGR parecem ser motivados pelo fato do autor perceber que quem as aceita não pode aceitar a MR.

A dilatação do tempo em campos gravitatórios é particularmente importante para demonstrar que os resultados da MR são inconsistentes com as observações. Seja por exemplo o caso de um corpo preso a uma mola oscilando horizontalmente. Este caso é considerado no MR [AK99m] e o resultado é que a freqüencia de oscilação é dada por

$$
\omega=\sqrt{\frac{k}{m_{g}}}
$$

onde $k$ é a constante elástica da mola. Se observa no MR que a diferença com o resultado na mecânica newtoniana é que na MR aparece $m_{g}$ (massa gravitacional) e não $m_{i}$ (massa inercial). O problema é quando se usa o resultado da Eq. (1) para afirmar [AK99m]:

Dobrando a quantidade de galáxias do universo, mantendo inalteradas a mola, a Terra e o corpo de prova, diminuiria a freqüência de oscilação em $\sqrt{2}$. Isto é equivalente a dobrar a massa inercial newtoniana do corpo de prova.

Isto é, se extrapola um resultado que na prática coincide com o da mecânica de Newton (e por isso não é importante) para o Universo todo! Qualquer sistema periódico é um relógio. Acontece que se usamos isso para calcular a diferença de tempos de 2 sistemas de molas, um na base e outro no alto de uma torre, a diferença de tempos segundo a MR é: zero! Segundo a MR teriamos

$$
\frac{\tau_{2}-\tau_{1}}{\tau_{1}}=\frac{N / \omega_{2}-N / \omega_{1}}{N / \omega_{1}} \equiv 0
$$

onde $N$ é o número de oscilações e $\omega_{1,2}$ são a freqüencias na base e no alto da torre. A identidade decorre da igualdade entre $\omega_{1}$ e $\omega_{2}$ uma vez que pela Eq. (1) as freqüências só dependem da massa gravitacional do corpo a qual é inalterável. ${ }^{11}$ No entanto como mencionado acima essa diferença dos relógios em campos gravitatórios já foi bem testada e está em acordo com as teorias da relatividade. De fato, experimentos que medem o desvio para o vermelho gravitacional usando relógios em torres e o sistema GPS, como comentado acima, confirmam a TRG [MI73b].

É interessante a afirmação com relação à dilatação do tempo necessária para explicar a chegada de píons e múons produzidos na atmosfera até a Terra [AK99g]:

o mesmo pode ser aplicado na experiência dos mésons. Ao invés de afirmar que o tempo anda mais lentamente para o corpo em movimento, nos parece mais simples e de acordo com a experiência afirmar que a meia-vida do méson depende ou dos campos eletromagnéticos a que foi exposto nesta situação ou ao seu movimento (velocidade ou aceleração) em relação ao laboratório e aos corpos distantes.

Acontece que a dilatação do tempo foi medida em circunstâncias diversas: em aceleradores, em experimentos em aviões e satélites, em experiências que medem o fator $g-2$ do múon, etc. Quais campos eletromagnéticos se aplicariam nestes casos? Mesmo na atmosfera, se existissem campos eletromagnéticos teriam outros efeitos por exemplo nas comunicações via satélite.

Vemos então, resumindo, que é a MR que não dá conta dos fatos observados como demostrado acima, um relógio na base de uma torre atrasa com relação a um relógio no topo da mesma devido à influência do campo gravitacional da Terra. A Eq. (2) mostra então que segundo a MR, molas oscilando horizontalmente na Terra não têm sua freqüência, dadas pela Eq. (1), alterada estejam elas na base ou no topo da torre. Tais osciladores seriam apenas um exemplo de relógios que não atrasariam devido ao campo gravitacional! A expressão dada na MR para a oscilação depende apenas da massa gravitacional e da constante da mola, que são conceitos primitivos em sua teoria e portanto não sofrem alteração das estrelas fixas.

Finalmente, a MR não é uma teoria de campos e portanto não prevê a emissão de ondas gravitacionais de maneira natural. Apesar dos grandes detectores terrestres de ondas gravitacionais ainda não estarem em funcionamento, ondas gravitacionais já foram indiretamente observadas em sistemas astrofísicos binários, como mencionado antes.

\section{Comentários finais}

O fato de uma teoria satisfazer ou não o Princípio de Mach (em qualquer uma de suas formulações) não pesa a favor ou contra a teoria, visto que não há qualquer experimento comprovando a validade dele, mesmo porque seria bastante difícil mover todas as estrelas do firmamento! A experiência do balde não pode ser considerada uma verificação experimental do princípio como é afirmado na MR.

Sabemos que a lei de Coulomb tem correções de origem quântica, mas nem por isso dizemos que a lei deve ser mudada, apenas reconhece-se que num determinado contexto (o átomo de hidrogênio, por exemplo) outros fatores são importantes. No caso da lei da gravitação

\footnotetext{
${ }^{11}$ Os autores agradecem G. E. A. Matsas discusões sobre este ponto. De fato, a Eq. (2) foi colocada pela primeira vez no debate realizado no IFGW-UNICAMP entre o autor da MR e Matsas.
} 
de Newton, que tinha sido testada para distâncias maiores que $1 \mathrm{~cm}$, pensava-se que poderiam ocorrer desvios para distâncias da ordem de $\mu \mathrm{m}$. Especula-se por exemplo, que, se existissem das dimensões espaciais extras, o potencial gravitacional de Newton seria substituído por uma expressão mais geral. Trata-se de uma proposta teórica, porém medidas recentes na escala de 200 $\mu \mathrm{m}$ não mostram desvios da lei de gravitação de Newton [HO01]. Mesmo que esse tipo de teorias venha a ser confirmada no futuro ainda assim continuaremos a usar o potencial de Newton em muitas das aplicações em distâncias de micrometros até milhares de quilômetros ou, dependendo da precisão, a TRG. Mesmo que desvios da lei da gravitação fossem um dia observados, cabe ressaltar que seriam oriundos de teorias consistentes na maioria dos aspectos com a TRG. Estas teorias têm de acrescentar outros ingredientes teóricos, como simetrias extras ou mais dimensões espaciais. Por outro lado, na eletrodinâmica quântica temos o deslocamento Lamb, efeito bem medido no átomo de hidrogênio, que implica numa correção ao potencial de Coulomb [HA84]. O que aprendemos com estes exemplos? A resposta é que temos de ter sempre em mente em que contexto uma modificação é feita numa lei básica.

Um aspecto que deve ser notado é que no prefácio da MR [AK99h] aparece o seguinte

Este livro é direcionado a físicos, matemáticos, engenheiros, filósofos e historiadores da ciência [...] Acima de tudo, é escrito para as pessoas jovens e sem preconceitos que têm interesse nas questões da física.

Deveria ser acrescentado e com pouco senso crítico, porque para aceitar a MR, depois das observações acima discutidas, é preciso, isto sim, ter preconceito a favor da mecânica relacional. Ainda no prefácio podemos ler [AK99i]

Após compreender a mecânica relacional entraremos num novo mundo, enxergando os mesmos fenômenos com olhos diferentes e sob uma nova perspectiva. É uma mudança de paradigma.

$\mathrm{O}$ autor se refere ao conceito de paradigma científico introduzido po T. Kuhn. Sinceramente, leitor, se você tem interesse nas questões da física, por acaso leu em algum lugar que Einstein, Heisenberg, Bohr, Dirac, Fermi, Pauli, e tantos outros conhecidos cientistas fizeram logo de início esse tipo de afirmação? Vamos além: esses autores escreveram livros sobre as suas teorias somente depois de alguns anos e quando a comunidade de físicos era majoritariamente a favor delas. Enfim, a verdadeira física nova só se percebe depois de certo tempo, mesmo para aqueles que a propuseram. Para Pais [PA95e]
A nova dinâmica contida nas equações relativistas generalizadas não foi completamente dominada, nem durante a vida de Einstein, nem no quarto de século que se seguiu à sua morte $[\cdots]$ nem mesmo num nível puramente clássico, ninguém pode hoje em dia gabar-se de ter um domínio completo do rico conteúdo dinâmico da dinâmica não linear designada por relatividade geral.

Apenas na ciência patológica as coisas são enganosamente claras de uma vez por todas. Aliás essa é, de fato, uma maneira de identificá-la. Os cientistas têm preconceitos, mas mesmo estes estão, na maioria dos casos, bem fundamentados. Agora sabemos, por exemplo, que o esquema de Copernico-Kepler-Galileu precisava de uma física nova, afinal formulada por Newton; que o que Boltzmann queria não era possível sem a mecânica quântica, que ainda não tinha sido descoberta. ${ }^{12}$

Assim, não é apenas pelos testes diretos, desde Michelson e Morley, que a relatividade é aceita como correta em certo domínio de fenômenos. Mais importante ainda é a consistência que ela trouxe para diversos domínios: astronomia, o sistema GPS, aceleradores de partículas, física sub-atômica, etc.

Como exemplo de ciência patológica podemos lembrar do caso Velikovsky [LA99, EB00]. Immanuel Velikovsky (1895-1879) propôs uma teoria astronômica em seu livro "Worlds in Collision". Ali ele dava argumentos sobre uma série de catástrofes ocorridas na Terra, uma delas teria provocado a abertura do Mar Vermelho para que os judeus vindos de Egito pudessem atravessar o mar. Ao ser questionado sobre a inexistência de outros registros além da Bíblia sobre esse tipo de catástrofe ele argumentava: "amnésia coletiva provocada pelas mesmas catástrofes"! São esses argumentos de natureza ad hoc que caraterizam a ciência patológica. Em particular, segundo o químico e prêmio Nobel I. Langmuir [ST00], a ciência patológica tem a seguinte caraterística (existem outras mas estas são mais relacionadas com a ciência experimental):

Teorias fantásticas contrárias às experiências e às críticas são enfrentadas com argumentos ad hoc improvisados no afã do momento.

As críticas no livro MR às TRE e TRG são desse tipo, o argumento de "campos magnéticos" para explicar a dilatação do tempo no caso dos raios cósmicos é característico desse tipo de argumentação ad hoc. Já foi discutido na seção anterior que isso não procede, porque a dilatação do tempo já foi medida em diversas situações e concorda bem com a TRE e a TRG. Ao obstinadamente negar estas teorias e tudo que elas implicam, o

\footnotetext{
${ }^{12}$ Mas seus métodos e princípios estavam corretos. Apenas a natureza não os realizava da maneira que ele acreditava.
} 
autor da MR é obrigado a recorrer a processos misteriosos e invocar fontes ainda não investigadas, mas que tenham, para o incauto leitor, uma aura de plausibilidade (efeitos novos, campos magnéticos desconhecidos, etc.). Por exemplo, no problema da precessão das órbitas dos planetas para fixar o valor observado é introduzido de forma ad hoc um parâmetro extra, $\xi$ [AK99k] que deve valer $\xi=6$ para concordar com as medições.

Por último e não menos importante, o que dizer com relação ao ensino de física no terceiro grau? Como apresentar para os estudantes, sob o ponto de vista da MR, afirmações como as que seguem (tomadas do respeitado e muito usado livro texto de Purcell [PU78b])

Vemos agora nos postulados da relatividade e suas implicações um sistema amplo, que envolve todas as leis da física e não apenas às do eletromagnetismo. Esperamos que qualquer teoria física completa seja relativisticamente invariante.

Ensinar-se-ia a um grupo de estudantes "sem preconceitos" que estas frases estão erradas? Que toda a física do Sec. XX também está? Não seria um crime deixar os estudantes nessa ignorância?

Mas não apenas no ensino no níveis de segundo e terceiro grau. Por exemplo, não é concebível que um biólogo, químico ou físico de outra especialidade, digamos de estado sólido ou ciência dos materiais, use ferramentas como luz síncroton e acredite que a eletrodinâmica de Weber ainda poderia ser considerada uma teoria rival àquela que permitiu a construção do aparelho que usa nas suas pesquisas.

Finalmente, gostaríamos de observar o seguinte. Mesmo se nos restringirmos à ciência normal ${ }^{13}$ podemos distinguir, numa mesma área, diferentes comunidades. A primeira divisão é pela especialização. Em geral uma comunidade tem uma ou várias revistas nas quais publica assuntos de um interesse que serve para definir essa comunidade. A maioria das referências usadas no livro MR estão em revistas onde não são usualmente encontrados trabalhos da ciência normal. Se alguém tem argumentos válidos de que as TRE e TRG estão erradas (esse aliás já seria um resultado impressionante) deveria publicar em revistas como Physical Review Letters. De nada adianta argumentar que essas revistas não publicariam, que têm preconceito etc. Isso mostra que as pessoas que apoiam os pontos de vista da MR pertencem a uma comunidade marginal.

Para terminar, esperamos ter deixado claro duas coisas: 1) não é apenas pelos testes diretos, desde Michelson e Morley, que as teorias da relatividade são aceitas como corretas em certo domínio de fenômenos. Mais importante ainda é a consistência que ela trouxe para diversos domínios: astronomia, aceleradores de partículas, física sub-atômica, o sistema GPS etc; 2) que a proposta da mecânica relacional [AK99] é errada e a resenha anterior [SO99] é, por isso, inconseqüente.

\section{Agradecimentos}

Agradecemos ao $\mathrm{CNPq}$ pelo auxilio financeiro parcial; a L. F. dos Santos pela leitura do manuscrito e a G. E. A. Matsas por úteis discussões sobre as teorias da relatividade.

\section{References}

[AK99] A. K. Assis, Mecânica Relacional, Centro de Lógica, Epistemologia e História da Ciência-UNICAMP, Campinas, 1998.

[AK99b] Ref. [AK99], p. 145.

[AK99c] Ref. [AK99], p. 178.

[AK99d] Ref. [AK99], p. 190.

[AK99e] Ref. [AK99], p. 200

[AK99f] Ref. [AK99], p. 205.

[AK99g] Ref. [AK99], p. 157.

[AK99h] Ref. [AK99], p. xviii.

[AK99i] Ref. [AK99], p. xvix.

[AK99j] Ref. [AK99], p. 179.

[AK99k] Ref. [AK99], p. 280.

[AK991] Ref. [AK99], p. 157.

[AK99m] Ref. [AK99], p. 254.

[AL88] C. Allègre, A Espuma da Terra, Gradiva, Lisboa, 1988.

[AN90] P. W. Anderson, On the nature of the physical laws, Physics Today, 43(12), 9 (1990).

[BO68] H. Bondi, Cosmology, Cambridge University Press, Cambridge, 1968; p. 31-33.

[BR01] H. N. Brown et al. (Muon $g-2$ Collaboration), hep$\mathrm{ex} / 0102017$

[DI00] M. Dine, The Utility of Quantum Field Theory, Palestra plenária no ICHEP 2000, Osaka, Japon; hep$\mathrm{ph} / 0010035$.

[EI05] A. Einstein, Ann. d. Phys. 17, 805 (1905). Tradução portuguesa em "Sobre a eletrodinâmica dos corpos em movimento", A. Einstein, H. Lorentz, H. Weyl e H. Minkowski, O Princípio da Relatividade, Fundação Calouste Gulbenkian, Lisboa, 1978; p. 47.

[EI05b] Ref. [EI05] p. 47-48.

[EI05c] Ref. [EI05] p. 72.

[EI17] A. Einstein, Kosmologische Betrachtungen zur allgemeinen Relativitätstheorie, Preuss. Akad. Wiss. Berlin, Sitzer., 142 (1917). Tradução portuguesa: Considerações cosmológicas sobre a teoria da relatividade geral, em Ref. [EI05], p. 141.

\footnotetext{
${ }^{13}$ Aqui usamos esse termo para rotular uma atividade de pesquisa que se publica em revistas com razoável parâmetro de impacto.
} 
[EB00] Immanuel Velikovsky, Encyclopædia Britannica Online.

[GE78] R. Geroch, General Relativity from A to B, University of Chicago Press, Chicago, 1978.

[HA84] F. Halzen e A. D. Martin, Quarks and Leptons, John-Willey, New York, 1984; p. 158.

[HE78] W. Heisenberg, A Parte e o Todo, Contraponto, Rio de Janeiro, 1996; p. 78.

[HE96] T. A. Herring, Sci. Am. 274, 32 (1996).

[HO82] G. Holton, Ensayos Sobre el Pensamiento Científico en la Época de Einstein, Alianza Editorial, Madrid, 1982; p. 177.

[HO82b] Ref. [HO82], p. 165.

[HO01] C. D. Hoyle, et al., Phys. Rev. Lett. 86, 1518 (2001).

[LA99] I. Lakatos e P. Feyerabend, For and Against Methods, University of Chicago Press, Chicago, 1999; p. $21-22$.

[MA83] A primeira edição alemã é de 1883. Aqui usaremos a versão inglesa: The Science of Mechanics, The Open Court Publishing Co., Chicago, 1942.

[MI73] C. W. Misner, K. S. Thorne e J. A. Wheeler, Gravitation, Freeman, New York, 1963; p.1095.

[MI73b] Ref. [MI73] p. 1055 e as referências ali citadas.

[NO93]

www. nobel.se/physics/laureates/1993/press.html

[NO00] http://ww.nobel.se/index.html

[PA95] A. Pais, "Sútil é o Senhor"... A Ciência e a Vida de Albert Einstein, Editora Nova Fronteira, Rio de Janeiro, 1995.
[PE98] R. Penrose, O Grande, o Pequeno e a Mente Humana, Editora UNESP, São Paulo, 1998; p. 37-38.

[PA95a] Ref. [PA95], p. 333.

[PA95b] Ref. [PA95], p. 335.

[PA95c] Ref. [PA95], p. 336.

[PA95d] Ref. [PA95], p. 340 .

[PA95e] Ref. [PA95], p. 314.

[PA95f] Ref. [PA95], p. 339.

[PU78] E. M. Purcell, Electricity and Magnetism. McGrawHill, Berkeley, 1978.

[PU78b] Ref. [PU78], p. 148.

[RE00] M. Rees, Apenas Seis Números: as forças profundas que controlam o universo, Rocco, Rio de Janeiro, (2000); p. 44.

[SA96] C. Sagan, O Mundo Assombrado Pelos Demonios, Companhia das Letras, São Paulo, 1996.

[SC49] P. A. Schipp, Albert Einstein, Philosopher-Scientist, Library of Living Philosophers, Evanston, 1949; p.67.

[SO99] D. S. L. Soares, Revista Brasileira de Ensino de Física, 21(4), 556 (1999).

[ST00] S. Stone, Pathological Science, hep-ph/0010295.

[WI79] C. M. Will, The confrontation between gravitation theory and experiment, em General Relativity, An Einstein Centenary Survey, S. W. Hawking e W. Israel (Eds.), Cambridge University Press, Cambridge, 1979.

[WI96] C.M. Will, Einstein estava certo? Colocando a Relatividade Geral à prova, Editora UnB, Brasília, 1996.

[WI79b] Ref. [WI79], p. 29. 